\title{
Comportamiento Superficial e Interfacial de Soluciones Acuosas Diluidas de Isómeros de Pentanodiol a 288.15K
}

\author{
Manuel. S. Páez ${ }^{(1) *}$, Armando Alvis ${ }^{(2)}$ y Guillermo Arrazola ${ }^{(2)}$ \\ (1) Universidad de Córdoba, Facultad de Ciencias Básicas e Ingeniería, Departamento de \\ Química, Carrera 6 No 76-103, Km 3, vía Cereté, Córdoba-Colombia (e-mail: \\ mspaezm@unal.edu.co) \\ (2) Universidad de Córdoba, Facultad de Ciencias Agrícolas, Departamento de Ingeniería de \\ Alimentos, Carrera 6 No. 76-103, Km 3, vía Cereté, Córdoba-Colombia. \\ * autor a quien debe ser dirigida la correspondencia
}

Recibido Abr. 28, 2010; Aceptado Jun. 08, 2010; Versión Final recibida Jun. 11, 2010

\begin{abstract}
Resumen
Se reportan medidas experimentales de tensión superficial para soluciones acuosas diluidas de 1,2-pentanodiol (12PD), 2,4-pentanodiol (24PD), 1,4-pentanodiol (14PD) y 1,5-pentanodiol (15PD) a $288.15 \mathrm{~K}$ como una función de la concentración, usando el método de ascenso capilar. Se discuten las interacciones que ocurren al interior y en la superficie de la solución y se interpreta este comportamiento superficial en términos de cantidades termodinámicas. Los resultados muestran la dependencia de la tensión superficial con la posición relativa de los grupos hidroxilos en la molécula de soluto y con el tamaño de la cadena alquílica expuesta al solvente.
\end{abstract}

Palabras clave: tensión superficial, isómeros de pentanodiol, centros de enlace, coeficientes de actividad

\section{Surface and interfacial behaviour of Dilute Aqueous Solutions of Pentanediol Isomers at 288.15 K}

\begin{abstract}
Experimental measurements of surface tensions are reported for diluted aqueous solutions of 1,2pentanediol (12PD), 2,4-pentanediol (24PD), 1,4-pentanediol (14PD) and 1,5-pentanediol (15PD) at $288.15 \mathrm{~K}$ as a function of the concentration, using the method of capillary rise. The interactions that occur in the solution and in the surface are discussed and the surface behaviour is interpreted in terms of thermodynamic quantities. The results show the dependence of the surface tension with the relative position of the hydroxylic groups in the solute molecule and with the size of the alquilic chain exposed to the solvent.
\end{abstract}

Keywords: surface tension, pentanediol isomers, binding sites, activity coefficients 


\section{INTRODUCCIÓN}

La tensión superficial de las mezclas líquidas es una propiedad que juega un papel importante en la interpretación del comportamiento de las soluciones acuosas, como en el caso del fenómeno hidrofóbico y otros procesos biológicos. Ella refleja cambios en las interacciones moleculares y de este modo puede ser aprovechada como un mecanismo para analizar, en particular, el comportamiento de las soluciones acuosas (Romero et al., 2007; Páez et al., 2009b).

La adsorción de sustancias orgánicas desde las soluciones acuosas es gobernada por varios factores. Por ello, el estudio de las mezclas diol-agua proporciona claves importantes para entender el efecto de la posición de grupos funcionales de las moléculas en la red de enlaces de hidrógeno tridimensional del agua. La razón es que en estas mezclas existe un delicado balance entre efectos "formadores de estructura" y "disruptores de estructura", es decir, ambos efectos hidrofóbico e hidrofílico juegan papeles igualmente importantes en ellos. Estudios previos han encontrado que éstas mezclas tienen efectos peculiares en sus propiedades fisicoquímicas en la región de bajas concentraciones (Romero y Páez, 2007).

La tensión superficial de las soluciones acuosas de 1,2-pentanodiol (12PD), 2,4-pentanodiol (24PD), 1,4-pentanodiol (14PD) y 1,5-pentanodiol (15PD) fue estudiada a bajas concentraciones de estos alcoholes debido a que la mayoría de los cambios en tensión superficial para sistemas no electrolíticos ocurren a muy baja concentración del soluto, mientras que los parámetros superficiales permanecen casi inalterados para altas concentraciones.

Finalmente este trabajo, es resultado del estudio sistemático desarrollado por el Laboratorio de Termodinámica de la Universidad de Córdoba, en aras a contribuir en el entendimiento del comportamiento fisicoquímico de las mezclas binarias de no electrólitos en medio acuoso a diferentes temperaturas, la primera de estas muestras ya fue publicada en esta misma revista en el año 2009 (Páez et al., 2009b). El objetivo de este trabajo consiste en mostrar cómo las propiedades superficiales de las soluciones acuosas de no electrólitos son reflejadas por las interacciones de las partes hidrofóbicas de las moléculas huésped adsorbidas en la interfase líquido-vapor. Los pentanodioles fueron escogidos como solutos porque son moléculas bastante pequeñas y su parte hidrofóbica es similar a la del n-butanol. Por otro lado, la existencia de dos grupos hidroxilos vecinos podría implicar interacciones específicas entre ellos, mientras cambia fuertemente la actividad superficial del soluto bajo investigación.

\section{MATERIALES Y METODOS}

Los reactivos usados son los siguientes: 1,2-pentanodiol, 96\% Aldrich, 2,4-pentanodiol, 98\% Aldrich 1,4-pentanodiol, 99\% Aldrich y 1,5-pentanodiol, 96\% Aldrich. Los cuatro alcoholes son reactivos analíticos y fueron destilados y desgasificados antes de su uso. El contenido de agua fue determinado en todos los casos por el método Karl Fisher. El agua fue doblemente destilada, tratada según la literatura y desgasificada antes de su uso (Weissberger, 1972). Todas las soluciones se prepararon por pesada usando una balanza OHAUS con una sensibilidad de $10^{-5} \mathrm{~g}$ en el rango mas bajo. Las concentraciones finales se corrigieron según el contenido de agua.

Las densidades de las soluciones fueron medidas usando picnómetros tipo Wood-Brusie con un volumen de bulbo de $80 \mathrm{~cm}^{3}$ y una incertidumbre de $\pm 5 \times 10^{-5} \mathrm{~g} \cdot \mathrm{cm}^{-3}$. Los picnómetros fueron calibrados con agua a la temperatura de trabajo, conforme a los datos de literatura (Riddick y Bunger, 1974).

La tensión superficial fue determinada a $288.15 \mathrm{~K}$, usando el método de ascenso capilar (Romero y Blanco, 1996) con un tubo capilar de $27.0 \mathrm{~cm}$ de longitud y $0.0653 \mathrm{~cm}$ de radio interno, determinado por calibración con agua a la temperatura seleccionada. Las medidas de altura se hicieron con un catetómetro Phylatex-Gerate con una división mínima de $1 \times 10^{-2} \mathrm{~cm}$. Todas las medidas se realizaron en un baño a temperatura constante controlada y las variaciones de temperatura en el mismo se registraron con un termómetro Fluke Hart Scientific de alta precisión con una incertidumbre de $\pm 0.005 \mathrm{~K}$. La tensión superficial de la solución $\sigma$ fue evaluada usando 
la ecuación de cálculo de Poisson-Rayleigh (1) en la que $\rho$ es la densidad de la solución, $r$ es el radio capilar, $h$ es la altura capilar y $g$ es la constante gravitatoria. Cada dato de tensión superficial se tomó como el promedio de tres medidas independientes. En todos los casos la desviación estándar es mucho menor que $0.3 \mathrm{mN} \mathrm{m}^{-1}$.

$$
\sigma=\frac{r \rho g}{2}\left(h+\frac{r}{3}-\frac{0.1288 r^{2}}{h}+\frac{0.1312 r^{3}}{h^{2}}\right)
$$

\section{RESULTADOS Y DISCUSIÓN}

En la figura 1, se correlacionan los resultados experimentales de la tensión superficial de las soluciones acuosas de los isómeros del pentanodiol como una función de la fracción molar a la temperatura de $288.15 \mathrm{~K}$. Se observa que el cambio de la tensión superficial es más grande para el 1,2-pentanodiol (12PD) y disminuye en el orden 2,4-pentanodiol (24PD), 1,4-pentanodiol (14PD) y finalmente se observa un comportamiento atípico para el 1,5- pentanodiol (15PD).

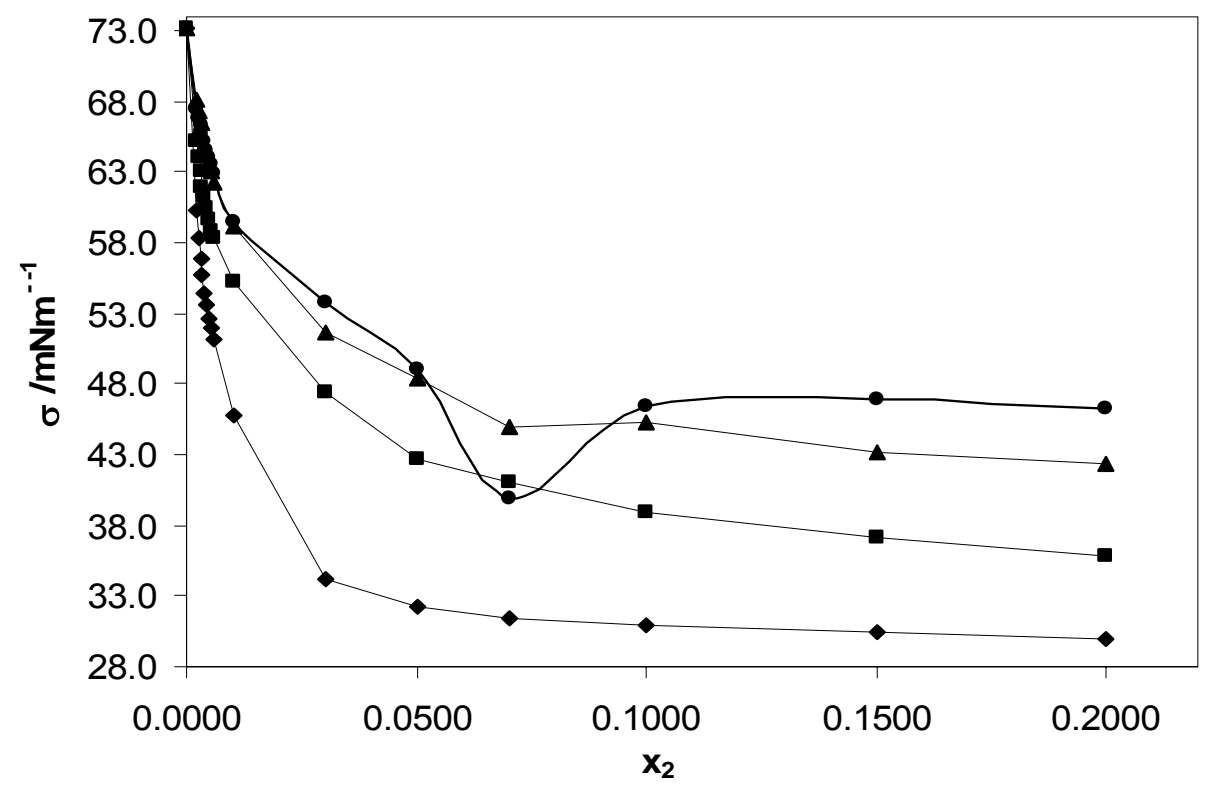

Fig. 1: Tensión superficial de soluciones acuosas diluidas de pentanodioles isoméricos: - 12PD, - 24PD, $\Delta$ 14PD y •15PD a 288.15K.

La dependencia con concentración de la tensión superficial para los pentanodioles considerados en este trabajo, muestra un comportamiento similar en la región muy diluida; la tensión superficial disminuye rápidamente cuando se incrementa la concentración del pentanodiol, lo cual es consistente con las tendencias encontradas para estos mismos sistemas a $283.15 \mathrm{~K}$ (Páez et al., 2009b). A concentraciones más altas la tensión superficial prácticamente se estabiliza comportándose como otros dioles y tensoactivos no iónicos (Habrdova et al., 2004; Connors y Wright, 1989; Hoke y Patton, 1992; Kwan y Rosen, 1980; Nakanishi et al.,1971; Romero y Páez, 2006; Zagórska et al., 1994; Hajji et al., 1989; Frindi et al., 1991; Romero et al., 2007).

Todos los sistemas aquí considerados siguen la misma tendencia reportada para otros glicoles, incluso el 1,5-pentanodiol mostró a la temperatura de $283.15 \mathrm{~K}$, el mismo comportamiento atípico reportado por Glinski (Glinski et al.,1999 ; Páez et al., 2009b).

En la tabla 1, se muestran las pendientes límites de los diferentes sistemas estudiados. De acuerdo a estos resultados el 1,2-pentanodiol exhibe el comportamiento hidrofóbico más pronunciado, debido a la influencia de la cadena alquílica con más alto dominio hidrofóbico expuesto al solvente acuoso, siguiendo el orden 2,4-pentanodiol, 1,4-pentanodiol y 1,5pentanodiol. 
Tabla 1: Pendientes limites de la tensión superficial para las soluciones acuosas de 1,2-pentanodiol; 2,4-pentanodiol; 1,4-pentanodiol y 1,5-pentanodiol

\begin{tabular}{ccccc}
\hline \multicolumn{5}{c}{$10^{-4}\left(\mathrm{~d} \sigma / \mathrm{dx}_{2}\right)_{\times 2 \rightarrow 0} / \mathrm{mNm}^{-1}$} \\
\hline $\mathrm{T}(K)$ & 12PD & 24PD & 14PD & 15PD \\
\hline 288.15 & $-0.28 \pm 0.02$ & $-0.193 \pm 0.007$ & $-0.159 \pm 0.002$ & $-0.111 \pm 0.002$ \\
\hline
\end{tabular}

En la tabla $2_{2}$ se presentan los parámetros resultantes y las constantes de enlazamiento o constante de centros de enlace $\mathrm{K}_{2}$ de acuerdo con el modelo de Connors (Connors y Wright., 1989). Las bases teóricas y la discusión detallada de este modelo pueden ser consultadas en la referencia previamente citada. Para todos los solutos considerados en este trabajo la constante de enlazamiento $\mathrm{K}_{2}$ es positiva como se esperaba. El más alto valor lo presenta el 1,2-pentanodiol, seguido por 2,4-pentanodiol; mientras que el 1,5-pentanodiol presenta un comportamiento inesperado, probablemente en virtud al comportamiento atípico de la tensión superficial. Este comportamiento confirma los resultados obtenidos por el método de la pendiente límite y puede ser atribuido a la solvatación hidrofóbica de los grupos alquílicos.

Tabla 2: Parámetros superficiales de Connors para soluciones acuosas de 1,2-pentanodiol (12PD), 2,4-pentanodiol (24PD), 1,4-pentanodiol (14PD) y 1,5-pentanodiol (15PD) a 288.15K.

\begin{tabular}{cccc}
\hline Soluto & $a$ & $b$ & $K_{2}$ \\
\hline 12PD & $0.995 \pm 0.006$ & $0.988 \pm 0.003$ & $199.0 \pm 2.2$ \\
24PD & $0.990 \pm 0.016$ & $0.870 \pm 0.008$ & $99.0 \pm 3.0$ \\
14PD & $0.988 \pm 0.006$ & $0.951 \pm 0.003$ & $82.3 \pm 0.8$ \\
15PD & $0.990 \pm 0.050$ & $0.910 \pm 0.030$ & $99.0 \pm 10.7$ \\
\hline
\end{tabular}

Los datos para las constantes $a$ y $b$ a esta temperatura muestran similitud con los reportados por Lavi y Marmur (2000) para alcoholes de cadena corta a 20,25 y $30^{\circ} \mathrm{C}$.

De los resultados hasta ahora mostrados es evidente que la influencia de la posición de los grupos $\mathrm{OH}$ es claramente reflejada en el comportamiento de las propiedades superficiales y las constantes de enlazamiento. Para dioles vecinos el comportamiento puede atribuirse a la fuerza del enlace de hidrógeno y a la capacidad para formar enlaces inter o intramoleculares de hidrógeno.

Para solutos superficialmente activos la concentración superficial de exceso $\Gamma_{2}$, puede ser considerada igual a la concentración superficial real sin que el error sea significativo. La concentración del tensoactivo en la interfase puede calcularse por consiguiente de datos de tensión superficial o interfacial usando apropiadamente la ecuación de Gibbs. Asumiendo comportamiento ideal, los resultados obtenidos para esta propiedad a $288.15 \mathrm{~K}$ se muestran en la figura 2 y 3.

Todas las gráficas de las isotermas de adsorción, muestran que el 1,2-PD, 2,4-PD y 1,4-PD exhiben un comportamiento interfacial similar; con excepción del 1,5-PD. De acuerdo con los resultados mostrados en la figura 2, se puede inferir que el 1,2-pentanodiol (12PD), 1,4pentanodiol (14PD) y 2,4-pentanodiol (24PD), se adsorben en la superficie, pero es el 1,2pentanodiol a concentraciones más bajas de este soluto en la fase líquida quien se acumula más rápidamente en la superficie, seguido en su orden por el 1,4-pentanodiol y el 2,4-pentanodiol; mientras que de acuerdo con la figura 3, el 1,5-pentanodiol (15PD) deja ver nuevamente su comportamiento atípico. La adsorción de estos solutos al interior del agua es debida fundamentalmente a fuerzas de dispersión.

El hecho de que la $\Gamma_{2}$ aumente con la fracción molar del alcohol, hasta alcanzar un máximo y luego disminuya (con excepción del 1,5-pentanodiol que lo hace de una forma irregular), podría sugerir que en la interfase se está presentando un fenómeno de crecimiento y destrucción de la red de enlaces de hidrógeno de los agregados del soluto formados a estas concentraciones, 
debido a que no se necesita ningún requisito para cubrir la superficie cuando se debilitan las interacciones moleculares en ese volumen.

Por otra parte, de acuerdo con Páez (2009a), la posición intermedia de un grupo hidrofílico más que su posición terminal en una cadena alquílica lineal o ramificada resulta en un incremento del área por molécula en la interfase liquido-aire (Páez et al., 2009a). Este razonamiento está en buen acuerdo con los resultados mostrados en la figura 4, ya que a mayor $\Gamma_{2}$ menor área molecular.

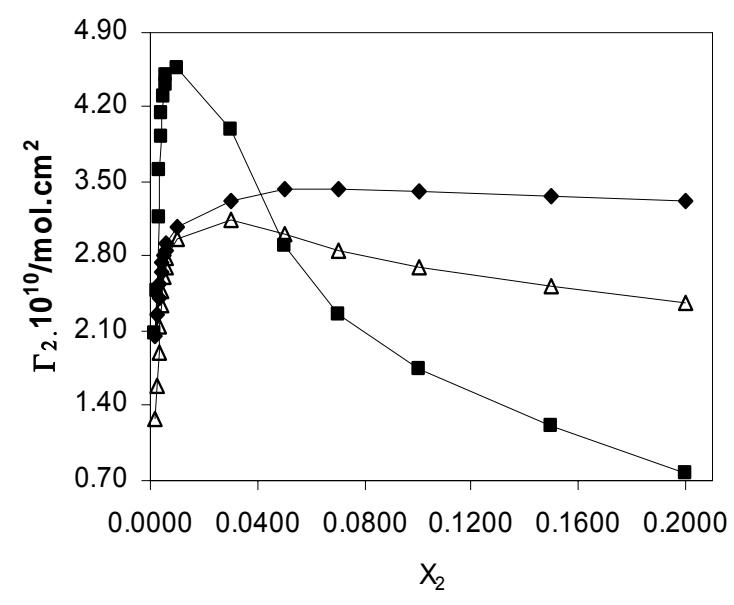

Fig. 2: Concentración superficial de soluciones acuosas diluidas de pentanodioles isoméricos: - 12PD, в 24PD, y $\Delta$ 14PD a 288.15K.

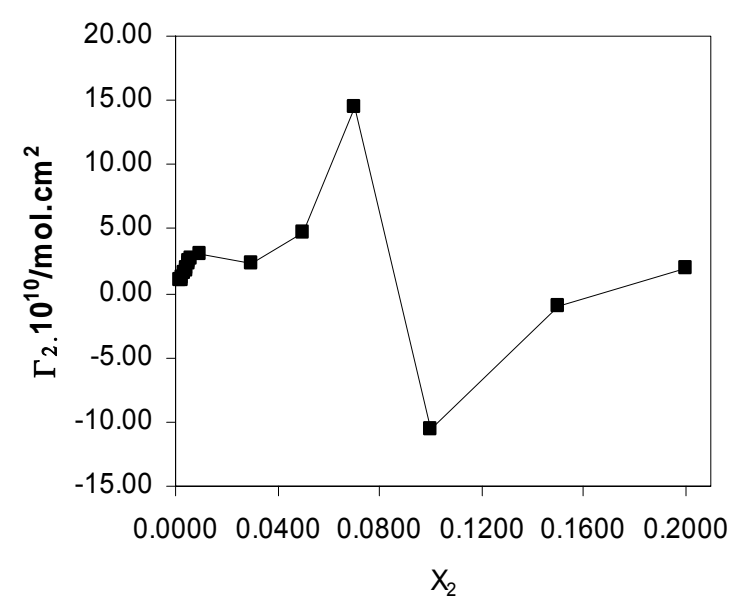

Fig. 3: Concentración superficial de soluciones acuosas diluidas de pentanodioles isoméricos: -15PD a 288.15K.

\section{CONCLUSIONES}

La tensión superficial de los solutos estudiados disminuye notablemente a bajas concentraciones, mientras que para las altas se estabiliza, con excepción del 1,5-pentanodiol, que presenta un comportamiento atípico. La influencia de la posición de los grupos $\mathrm{OH}$ es claramente reflejada en el comportamiento de las propiedades superficiales, las constantes de enlazamiento y las concentraciones superficiales. El signo negativo de la pendiente límite confirma el comportamiento hidrofóbico de los sistemas estudiados.

Se discuten las interacciones que ocurren al interior y en la superficie de la solución. Se establece que la disminución en la tensión superficial es resultado del aumento de la actividad del soluto en el seno de la solución. El área ocupada por estos solutos en la región superficial parece estar dominada por los grupos hidrofílicos hidratados.

\section{AGRADECIMIENTOS}

Loa autores agradecen a la Universidad de Córdoba por el apoyo prestado para la realización de este trabajo.

\section{REFERENCIAS}

Connors, K. A. y J. L. Wright; Dependence of surface tension on composition of binary aqueousorganic solutions, Anal. Chem.: 61(3), 194-198 (1989).

Frindi, M., B. Michels y R. Zana; Ultrasonic absorption studies of surfactant exchange between micelles and bulk phase in aqueous micellar solutions of nonionic surfactants with short alkyl chains. 1,2-Hexanediol and 1,2,3-octanetriol, J. Phys. Chem.: 95(12), 4832-4837 (1991). 
Glinski, J., G. Chavepeyer y J. K. Platten; Untypical surface propierties of aqueous solutions of 1,5pentanediol, Colloids and Surfaces A.: 162(1-3), 233-238, (1999).

Habrdova, K., S. Hovorka, L. Bartovska.; Concentration dependence of surface tension for very dilute aqueous solutions of organic nonelectrolytes, J. Chem. Eng. Data.: 49(4), 1003-1007 (2004).

Hajji, S. M. y otros cinco autores; A comparative study of hexanediol-1,2 and octanetriol-1,2,3 in aqueous solutions by different physical techniques, J. Phys. Chem.: 93(12), 4819-4824 (1989).

Hoke, B. C. y E. F. Patton; Surface tensions of propylene glycol + water, J. Chem. Eng. Data.: 37(3), 331-333 (1992).

Kwan, C. C. y M. J. Rosen; Relationship of structure to properties in surfactants. 9. Syntheses and properties of 1,2- and 1,3-alkanediols, J. Phys. Chem.: 84(5), 547-551 (1980).

Lavi, P. y A. Marmur; Adsorption Isotherms for Concentrated Aqueous-Organic Solutions (CAOS), J. Colloid Interface Sci.: 230(1), 107-113 (2000).

Páez, M. S., E. E. Villegas y A. Alvis; Propiedades de Superficie en Soluciones Acuosas Diluidas de Alcoholes con Tres Carbonos, Inf. Tecnol.: 20(1), 83-90 (2009A)

Páez, M. S., G. J. Borja y A. Alvis; Tensión Superficial de Soluciones Acuosas Diluidas de Isómeros de Pentanodiol a 283.15 K, Inf. Tecnol.: 20(2), 47-53, (2009 $)$.

Nakanishi, K., T. Matsumoto y M. Hayatsu; Surface Tension of Aqueous Solutions of Some Glycols, J. Chem. Eng. Data.: 16(1), 44-45 (1971).

Riddick, J. A. y W. B. Bunger; Techniques of Chemistry Vol II. Wiley-Interscience, New York (1974)

Romero, C. M. y L. H. Blanco; Tópicos en Química Básica, Academia de Ciencias Físicas, Exactas y Naturales, $1^{\text {a }}$ edición, 191-192. Guadalupe, Bogotá, Colombia (1996)

Romero, C. M. y M. S. Páez; Surface tension of aqueous solutions of alcohol and polyols at 298,15K, Phys. Chem. Liq.: 44(1), 61-65 (2006).

Romero, C. M. y M.S. Páez; Volumetric Properties of Aqueous Binary Mixtures of 1-Butanol, Butanediols, 1,2,4-Butanetriol and Butanetetrol at 298.15K, J. Solution Chem.: 36, 237-245, (2007).

Romero, C. M. y otros cuatro autores; Effect of temperature on the surface tension of diluted aqueous solutions of 1,2-hexanediol, 1,5-hexanediol, 1,6-hexanediol and 2,5-hexanediol, Fluid Phase Equilib.: 258(1), 67-72 (2007).

Weissberger, A. (Ed.); Physical Methods of Chemistry, Vol. 1 Part $4,4^{\text {th }}$ ed. Wiley New York (1972).

Zagórska, I., Z. Koczorowski y S. Trasatti; Surface and interfacial behaviour of isomeric butanediols, J. Electroanal. Chem.: 366(1-2), 211-218 (1994). 